\title{
Safety Analysis and System Design of Lithium Iron Phosphate Battery in Substation
}

\author{
Zhang Fang ${ }^{1}$ Li Junming ${ }^{2}$ Yu Xiaochen ${ }^{3}$ Su Hainan $^{3}$ Yu Xin ${ }^{3}$ Pang Jing ${ }^{3 *}$ Xie Hongxu ${ }^{3}$ \\ ${ }^{1}$ Sate Grid Dandong Electric Power Supply Company, Dandong, Liaoning, 118000, China \\ ${ }^{2}$ Yantai Haibo Electrical Equipment Co., Ltd, Yantai, Shandong, 264001, China \\ (Note: The authors are ranked as follows: Zhang Fang is the first author, Li Junming is the second author, and Yu Xiaochen, Su Hainan, \\ Yu Xin, Pang Jing, and Xie Hongxu are the co-third authors)
}

\begin{abstract}
This paper discusses the safety protection design of lithium iron phosphate batteries based on the technical characteristics of lithium iron phosphate batteries. Combined with the current background of the application of lithium iron phosphate batteries in substations, the system design of lithium iron phosphate batteries is discussed from many aspects. It focuses on how to ensure its safety in order to improve the application effect of lithium iron phosphate batteries in substations.
\end{abstract}

\section{Introduction}

In recent years, with the continuous improvement of the national power grid construction level, concepts such as environmental protection, energy saving and emission reduction have become important goals for the development of power grid construction. As a result, lithium iron phosphate batteries with unique advantages have been more and more widely used in substations. However, there are still some unavoidable risk problems in the working process of lithium iron phosphate batteries. Therefore, how to ensure the safe application of lithium iron phosphate batteries in substations is still an important research, which is necessary to further analyze its safety and system design.

\section{Technical characteristics of lithium iron phosphate battery}

\subsection{Basic working principle}

The basic components of lithium iron phosphate batteries are the same as other types of batteries. They are composed of positive and negative electrodes, separators, electrolyte, and casing. Among them, the positive and negative electrodes are composed of various active materials. These active materials participate in a series of chemical reactions in the charge and discharge process, and they are also the core of the battery [1]. When the battery is discharged, the positive and negative ions inside the battery move directly through the external circuit to form current. The negative electrode loses electrons and is oxidized. The positive electrode that receives electrons is reduced, thereby converting chemical energy into electrical energy. During the charging process, the chemical reaction that occurs on the electrode is exactly the opposite of the former.

Generally, lithium iron phosphate batteries use lithium iron phosphate as the positive electrode material. Elemental carbon as the negative electrode material are immersed in an organic solvent of lithium hexafluorophosphate. The flow of lithium ions between the positive and negative electrodes is used to generate current.

\subsection{Technical characteristics of lithium iron phosphate battery}

The basic technical characteristic parameters of lithium iron phosphate batteries are shown in table 1 .

Table 1. Basic parameters of lithium iron phosphate batteries

\begin{tabular}{cc}
\hline Item & Parameter \\
\hline $\begin{array}{c}\text { Maximum capacity } \\
\text { (monomer) }\end{array}$ & $500 \mathrm{Ah}$ \\
Maximum capacity (scale & $400 \mathrm{Ah}$ \\
monomer) & \\
Standard discharge voltage & $3.2 \mathrm{~V}$ \\
Final discharging voltage & $2.5 \mathrm{~V}$ \\
Energy density per unit & $190 \mathrm{kWh} / \mathrm{m}^{3}$ \\
volume & \\
Weight of monomer & $13.7 \mathrm{~kg}$ \\
Charge/discharge efficiency & $97.0 \%$ \\
\hline
\end{tabular}

\subsection{Technical characteristics of lithium iron phosphate battery}

The basic technical characteristic parameters of lithium iron phosphate batteries are shown in table 1 .

\footnotetext{
*Corresponding author: tg667788@xzcstudio.com
} 
In addition to the basic characteristics mentioned above, compared with other batteries, lithium iron phosphate batteries have smaller internal resistance and self-discharge characteristics. In the case of standing still, the power loss is relatively slow. In the charging process, constant current charging is the mainstay in the initial stage until the battery discharge voltage reaches a stable value, then constant voltage charging is performed. During the discharge process, the output voltage of the lithium iron phosphate battery is relatively stable, and it can achieve high rate discharge [2].

According to relevant data, the service life of lithium iron phosphate batteries has obvious advantages compared with traditional lead-acid batteries. After 5000 cycles of charging and discharging, it can still maintain nearly $90 \%$ of the initial power, so its practical application value is higher. Not only that, because the raw materials used in the preparation of lithium iron phosphate batteries are generally non-toxic and harmless, some of the materials are even directly derived from the components of former waste batteries. It can be seen that the application of lithium iron phosphate batteries fully meets the requirements of today's environmental protection and sustainable development.

\section{Safety protection analysis of lithium iron phosphate battery}

\subsection{Active safety protection}

Active safety protection design can be divided into the following aspects. One is the design of the battery body. During the charging and discharging process of the lithium iron phosphate battery, it is inevitable that a certain amount of heat will be generated. For this reason, the thermal stability of the electrode and electrolyte materials is the primary consideration. In general, some chemical additives should be appropriately added to prevent the battery from deflagration due to problems such as overcharge, short circuit, sudden temperature change, drop or impact by sharp objects.

The second is the design of the battery casing. According to the actual needs, it can choose steel, aluminum, organic polymer materials and other shell materials, and combine several batteries into a battery pack in an integrated design, and then carry out the final design of the shell to reduce external factors impact on the battery pack. On the basis of ensuring a reasonable structure of the battery casing, the aesthetics of the casing can be further considered.

The third is the design of the charger device. In this link, on the one hand, it is necessary to ensure that the voltage and current of the charging device can be stabilized. On the other hand, it can make sure that the charger is protected from over-current and over-voltage. To this end, corresponding monitoring devices and sensors can be installed to implement corresponding safeguard measures [3].

\subsection{Passive safety protection}

The passive safety protection of battery is mainly reflected in the following three aspects.

One is the flame-retardant and fire-resistant design. In this design link, adding a flame retardant coating to the inner wall of the battery box is a key content, which helps prevent the further spread of battery combustion and gains enough time to deal with the problem. At present, in order to ensure the effectiveness of the flame-retardant and fire-resistant design, technicians usually choose the design method of spraying a double-layer flame retardant coating.

The second is a dedicated automatic fire extinguishing device. This device mainly detects the battery cell temperature and smoke concentration. When it is detected that the cell temperature and smoke concentration reach the critical point of danger, it will be determined that the battery has caught fire, and the automatic fire extinguishing device will be activated immediately to extinguish the fire, and a warning message will be issued. In the special automatic fire extinguishing device, the high-efficiency and high-pressure fire extinguishing agent is mainly used, which can not only effectively reduce the temperature of the cell inside the battery, but also repair the battery diaphragm through chemical inhibition to avoid the occurrence of free radical reactions, thereby preventing the fire of the battery.

The third is a smoke detection and alarm device, which can also monitor the internal condition of the battery in real time. When the concentration of smoke particles or nitrogen oxides collected exceeds the critical point, it will be judged that the battery is on fire, and a warning will be issued in time to remind the staff to evacuate the scene in time. At present, in order to improve the detection efficiency and avoid false alarms, most of the device is equipped with a suction device [4].

\subsection{Safety protection code}

In order to ensure that lithium iron phosphate batteries can be used safely, the following requirements must be observed. The first is to check the appearance of the battery regularly. If it is found to be swollen or damaged, it should be stopped and replaced immediately. The second is that the battery must be disassembled by professionals. The third is to ensure that the battery is used in a reasonable environment and avoid using the battery in extreme environments.

\section{System design of lithium iron phosphate battery in substation application}

\subsection{Safety analysis}

At present, the main factor restricting the application of lithium iron phosphate batteries in substations is the safety of lithium iron phosphate batteries. There are too 
many factors leading to safety accidents in lithium iron phosphate batteries. Especially in the case of overcharge, a large amount of lithium metal will be deposited on the negative electrode, which will cause the temperature of the solvent to rise and cause safety problems. Other factors such as vibration, short-circuiting and squeezing are hardly generated because the battery is located in a dedicated battery room. Therefore, how to solve the problem of battery overcharge and over-discharge is the key to ensuring the safety of lithium iron phosphate battery packs.

To ensure that the voltage and current meet the requirements, multiple battery cells are usually connected in series to form a battery pack. This will cause some of the cells to be overcharged due to the difference in voltage parameters between the battery cells, which will lead to a rapid reduction in the service life of the entire battery pack, and even cause the battery pack to catch fire and explode in severe cases. Therefore, it is essential to adopt balanced measures. At present, avoiding overcharging is mainly based on the resistance discharge function of the battery management system. However, it will cause the battery management system itself to be in a heating state, which brings new safety risks. For this reason, new charging control measures are still issues that need to be considered.

At the same time, due to the large number of lithium iron phosphate batteries connected in series, the probability of safety risks will increase. Therefore, the application of large-capacity lithium iron phosphate batteries is still in its infancy. Moreover, the safety of large-capacity lithium iron phosphate batteries is relatively poor. For this reason, the simple series connection is not conducive to ensuring the safety of lithium iron phosphate batteries. How to design a series-parallel combination topology is a problem that needs to be further explored [5].

\subsection{Battery capacity selection}

At present, in the process of substation application, the selection of lithium iron phosphate battery capacity is still a difficult problem. In the current actual work, more reference is made to the data of lead-acid batteries. The discharge parameters of current mainstream lithium iron phosphate batteries and lead-acid batteries are shown in table 2 .

Table 2. Comparison of galvanostatic discharge data between lithium iron phosphate and lead acid batteries

\begin{tabular}{lllll}
\hline Time $(\mathrm{min})$ & 15 & 20 & 30 & 60 \\
\hline $\begin{array}{l}\text { Lithium iron phosphate } \\
\text { battery (final voltage }\end{array}$ & 370 & 280 & 190 & 100 \\
$\begin{array}{l}2.5 \mathrm{~V}) \text { /A } \\
\text { Lead-acid cell ( final } \\
\text { voltage } 1.65 \mathrm{~V}) \text { /A }\end{array}$ & 175 & 135 & 100 & 6 \\
\hline
\end{tabular}

\subsection{Topology of DC systems}

At present, due to the large-scale production of lithium iron phosphate battery monomer capacity is only about
400Ah, and many substations require a single battery capacity of 500Ah or even higher. Therefore, the limiting factor of the monomer capacity is extremely obvious, and the method of topology optimization must be adopted. At present, the commonly used topology is mostly a combination of series and parallel. It can connect each battery pack in parallel and in series with the master control device. After adopting this topology, due to the differences in the parameters of each lithium iron phosphate battery cell, the battery circulation problem is also inevitable. The battery circulation problem will significantly reduce the service life of the battery pack. For this reason, how to solve the battery circulation problem is a key point that needs to be considered first.

At present, in order to solve the battery circulation problem, it is mainly solved by the following two methods. The first is to strengthen the detection of the performance parameters of the battery cells, give priority to selecting a batch of battery cells with better parameter consistency for application, and optimize the design of the battery series-parallel combination on this basis. The second is to add a DC/DC module to the lithium iron phosphate battery pack to adjust the output of the battery cells so that the output of each battery cell is consistent. From the current application effect, these two methods have achieved certain results, but it still needs to be further improved. The future research direction will still focus on how to optimize the topological structure of the DC system to further improve its efficiency.

\subsection{Charging control principle and seamless power supply principle}

In the current lithium iron phosphate battery pack, in order to adjust the charging voltage and current parameters reasonably, IGBT devices are usually used for charging control. When the external power supply parameters do not meet the requirements, the voltage and current input to the battery pack can be intelligently adjusted to avoid safety issues by excessive high current. When multiple battery packs are charged at the same time, the control system adjusts the conduction rate of the IGBT device, and stops charging the battery pack to avoid damage to the battery pack.

The principle of seamless power supply means that the lithium iron phosphate battery pack can supply real-time power to the load unit. At the same time, during the non-charging process, if there is a significant voltage difference between the battery packs, the communication between the battery packs will be cut off to avoid circulating currents between the battery packs. This kind of circuit can also be called diode parallel equivalent circuit, which can ensure that the voltage and current of each battery pack are in a balanced state.

\section{Conclusion}

In general, because lithium iron phosphate batteries have many advantages such as high energy density and low loss, it is a general trend to replace traditional batteries in substation applications. But from the current point of 
view, its safety issue is still a key factor restricting its further application. For this reason, it is still necessary to optimize and innovate in the battery body, battery casing, battery management and battery pack structure to solve a series of existing problems and provide technical support for the effective application of lithium iron phosphate batteries in substations.

\section{Acknowledgment}

Project Research: Application Research on New Battery Based on Energy Saving and Environmental Protection Material in Operation and Maintenance of DC Power Supply System in Substation

\section{References:}

1. Huang Junwei, Lou Panpan, Zhai Sijie, Li Longguang, Zhao Ruixia. Research on the safety protection technology of lithium iron phosphate batteries for station use [J]. Electronic World, 2020(17): 59-60.

2. Lin Bin, Miao Yan, Li Bin, Hu Xiaohui, Zheng Qiuyun. Research on the charging and discharging strategies of lithium iron phosphate DC system in substation [A]. Fujian Electrical Engineering Society. Fujian Electrical Engineering Society 2019 Academic Annual Conference Award-winning Proceedings [C].: Fujian Society of Electrical Engineering, 2020: 5.

3. Liu Han, Miao Yan, Li Hong, Xie Yi, Chen Bin. Research on the design and application of a new generation of mobile emergency power supplies in substations[J]. Communication World, 2019, 26(06): 138-139.

4. Mei Chenglin, Zhao Wei. Safety analysis and system design of lithium iron phosphate batteries in substations $[\mathrm{J}]$. Electrical Technology, 2019, 20(06): 70-73.

5. Pang Bo. Design of substation energy storage configuration scheme and its economic analysis [A]. China Electric Power Research Institute. Proceedings of 2018 Smart Grid New Technology Development and Application Seminar [C]. China Electric Power Research Institute: Editorial Department of Computer Engineering and Applications, 2018: 7. 